\title{
Oxidized low-density lipoprotein upregulates microRNA-146a via JNK and NF-кB signaling
}

\author{
ZHENWEI LI* , SHUAI WANG ${ }^{*}$, WENTING ZHAO, ZEWEI SUN, HUI YAN and JIANHUA ZHU \\ Department of Cardiology, First Affiliated Hospital, School of Medicine, \\ Zhejiang University, Hangzhou, Zhejiang 310003, P.R. China
}

Received February 3, 2015; Accepted November 24, 2015

DOI: $10.3892 / \mathrm{mmr} .2015 .4729$

\begin{abstract}
Increasing evidence suggested the involvement of microRNA (miR)-146a in the pathogenesis of multiple diseases, including atherosclerosis, bacterial infection and cancer. However, the mechanism by which miR-146a is regulated in macrophages exposed to oxidized low-density lipoprotein (oxLDL) has remained elusive. The present study aimed to explore the molecular pathway of miR-146a regulation in response to oxLDL. Human THP-1 macrophages were pre-treated with small interfering RNA specific for scavenger receptors or with pharmacological inhibitors prior to oxLDL administration. A filter plate screening assay was performed to identify oxLDL-inducible transcription factors that bind to the miR-146a promoter. The exact binding sites were mapped by chromatin immunoprecipitation. The effects of miR-146a on markers of macrophage maturation were studied by flow cytometry. The results revealed that miR-146a expression was deceased when c-jun N-terminal kinase (JNK) or nuclear factor $(\mathrm{NF})-\kappa \mathrm{B}$ signaling was inhibited. By forming a complex with c-jun, which was promoted by oxLDL, the NF- $\mathrm{NB}$ sub-unit p65 facilitated the binding of c-jun to the miR-146a promoter to trigger transcriptional activation. miR-146a negatively regulated macrophage maturation by reducing the expression of CD86 and CD80. The present study demonstrated that oxLDL positively regulates miR-146a via the JNK and $\mathrm{NF}-\kappa \mathrm{B}$ pathways in macrophages, and that miR-146a inhibits inflammatory activation.
\end{abstract}

Correspondence to: Mr. Jianhua Zhu, Department of Cardiology, First Affiliated Hospital, School of Medicine, Zhejiang University, 79 Qingchun Road, Hangzhou, Zhejiang 310003, P.R. China E-mail: zjh_john@medmail.com.cn

*Contributed equally

Key words: microR-146a, oxidized low-density lipoprotein, c-Jun $\mathrm{N}$-terminal kinase, nuclear factor $\kappa \mathrm{B}$

\section{Introduction}

Atherosclerosis is a chronic lipid-associated inflammatory disease (1). Hyperlipidemia triggers multiple metabolic disturbances and has been considered as an important pathogenic factor. Oxidized low-density lipoprotein (oxLDL) engulfed by macrophages activates a variety of genes and the expression of microRNAs (miRNAs/miRs). miRNAs are a class of single-stranded nucleotides of $\sim 22$ bases in length, which induce the formation of silencing complexes in a sequence-specific manner, leading to translational repression of target genes (2). miRNAs are involved in numerous cellular biological activities associated with pathological processes, including cardiogenesis, oncogenesis, immunological diseases and hematopoietic differentiation (3). miR-146a modulates innate immunity (4), neoplasia (5) and infection (6) by targeting Toll-like receptor (TLR) signaling. Previous studies have indicated that miR-146a significantly controls diverse aspects of autoimmune diseases, including systemic lupus erythematosus (7), rheumatoid arthritis (8-10) and psoriasis (11). In spite of several studies that established the involvement of miR-146a in immune-inflammatory diseases, the underlying mechanism of its regulatory function has largely remained elusive. A previous study by our group showed that oxLDL activates miR-146a expression, which in turn stimulates various inflammatory factors (12). The present study investigated the regulation of miR-146a by oxLDL in cells of the immune system with the aim of identifying novel therapeutic targets for the treatment of atherosclerosis. Due to its known association with atherosclerosis, the THP-1 human macrophage cell line was used as a model (13).

\section{Materials and methods}

Cell culture. The THP-1 human monocytic cell line was a gift from the State Key Laboratory for Diagnosis and Treatment of Infectious Diseases (Zhejing University, Hangzhou, China). The cells were cultured in RPMI 1640 medium (Gibco; Thermo Fisher Scientific, Waltham, MA, USA) containing $10 \%$ fetal calf serum (Gibco). Prior to each experiment, the cells were treated for $24 \mathrm{~h}$ with $100 \mathrm{nmol}$ phorbol myristate acetate (Sigma-Aldrich, St. Louis, MO, USA) to acquire a macrophage-like adherent phenotype. Pre-treatment with chemical inhibitors, including c-jun N-terminal kinase (JNK) 
inhibitor SP600125, p38/mitogen-activated protein kinase (MAPK) inhibitor SB203580, MAPK kinase inhibitor UO126 or nuclear factor (NF)- $\kappa \mathrm{B}$ inhibitor pyrrolidine dithiocarbamate (PDTC) (all from Beyotime Institute of Biotechnology, Inc., Haimen, China) was performed for $30 \mathrm{~min}$ prior to addition of $40 \mu \mathrm{g} / \mathrm{ml}$ oxLDL (Guangzhou Yiyuan Biological Technology Co., Ltd., Guangzhou, China). The cells were harvested after $24 \mathrm{~h}$ of oxLDL treatment.

Small interfering (si)RNA transfection. The cells were placed in serum-free complete medium $30 \mathrm{~min}$ prior to transfection. siRNAs against lectin-like oxLDL receptor-1 (LOX-1; GTA TATGCTAATTGCTTTTA), macrophage scavenger receptor class A (SRA; CTAGAATTTGTATTGCTACA), CD36 (GCTGAGAATAAGCAGCAATA), c-jun (CCTGGCAAA TCAACAAGGTA), p65 (CCTTCTGCCTCCCAGGTTCA) (all from Baiao Biotech Products Corp., Tianjin, China) or miR-146a mimic/mimic-control or miR-146a inhibitors/inhibitor-control (Ruibo Biotech Products Co., Guangzhou, China) were transfected using the Lipofectamine RNAiMAX transfection reagent (Thermo Fisher Scientific, Inc.) following the manufacturer's recommendations. A total of $2 \mu 1$ siRNA at a final concentration of $50 \mathrm{nM}$ or $2 \mu \mathrm{g}$ plasmids, were mixed with $3.3 \mu \mathrm{l}$ transfection reagent in RNase-free tubes containing $200 \mu \mathrm{l}$ serum-free medium. After $15 \mathrm{~min}$, the mixture was added into the cell culture plate, and following $24 \mathrm{~h}$ the cells were harvested.

p65 overexpression plasmids transfection. The cells were cultured in serum-free medium for $30 \mathrm{~min}$ prior to transfection. A total of 2 ug p65 overexpression plasmids (pCMV-p65) or 2ug blank plasmids (pCMV-basic; OriGene Technologies, Inc., Rockville, MD, USA) were mixed with $5 \mu 1$ p3000 (Thermo Fisher Scientific, Inc.) in $200 \mu \mathrm{l}$ serum-free medium. After $5 \mathrm{~min}$, the mixture was added into another $205 \mu \mathrm{l}$ serum-free medium containing $5 \mu \mathrm{l}$ Lipofectamine 3000 (Thermo Fisher Scientific, Inc.). At 10 min later, the mixture was added into the cell culture plate. The cells were harvested following 24 hours.

Reverse transcription-quantitative polymerase chain reaction (RT-qPCR) analysis of miRNA. Total RNA was isolated using the miRcute miRNA isolation kit (Tiangen Biotech Co., Ltd., Shanghai, China). Subsequently, cDNA was generated using the PrimeScript miRNA RT Reagent kit (Takara Biotechnology Co., Dalian, China). The PCR reaction was performed using SYBR premix Ex taq (Takara) and the ABI PRISM 7500 Sequence Detection System (Thermo Fisher Scientific, Inc.). RT-qPCR consisted of 40 cycles of $95^{\circ} \mathrm{C}$ for $5 \mathrm{sec}, 60^{\circ} \mathrm{C}$ for $30 \mathrm{sec}$ and $95^{\circ} \mathrm{C}$ for $30 \mathrm{sec}$. The primer sequences were as follows: miR-146a, forward 5'-GGCTGAGAACTGAATTCC AUGGGTT-3' and reverse poly-T; and U6, forward 5'-CCG CACTTGATACGGTTCCT-3' and reverse 5'-CCAGGCTGA TCTATCCCACTG-3'. Measurement of U6 RNA was used as an endogenous control. Values were normalized to U6 using the $2^{-\Delta \Delta} \mathrm{Cq}$ method.

Dual luciferase reporter assay. Genomic DNA was extracted from THP-1 cells using Genome DNA Extract kit (Kangwei Biotech Co., Hangzhou, China). A segment of the miR-146a promoter, encompassing 2,000 bp upstream of the transcription initiation site, was amplified by PCR which consisted of 40 cycles of $95^{\circ} \mathrm{C}$ for $10 \mathrm{sec}, 55^{\circ} \mathrm{C}$ for $15 \mathrm{sec}$ and $72^{\circ} \mathrm{C}$ for $30 \mathrm{sec}$. The PCR product was gel-purified and sub-cloned within the HindIII and SacI restriction sites of the pGL3-basic Firefly luciferase reporter vector (Promega Corp., Madison, WI, USA), using restriction enzymes (Sangon Biotech Co., Ltd., Shanghai, China). A reporter plasmid containing a mutated version of the miR-146a promoter was also constructed. Each reporter plasmid $(2 \mu \mathrm{g})$ was co-transfected with a Renilla luciferase plasmid (20 ng) into THP-1 cells by using Lipofectamine 3000 (Thermo Fisher Scientific, Inc.). Following transfection for $48 \mathrm{~h}$, cells were washed twice and lysed with lysis buffer (Beyotime Institute of Biotechnology) containing $20 \mathrm{mmol}$ Tris (pH 7.5). Firefly luciferase activity was measured using the dual-luciferase reporter assay system (Promega Corp.). Relative luciferase activity of the reporter vector was obtained by normalization to the Renilla control luciferase activity. The primers used to amplify the miRNA-146a promoter were as follows: 2,000 bp-promoters, forward, 5'-ATCTGA CTGACTGGGGTCATCG-3' and reverse, 5'-GTCGATCGT ACGTACGAATGCG-3'; and 1,000 bp-promoters, forward, 5'-GACTACAATCACGAACTGCCA-3' and reverse, 5'-CCA GGCTGATTCATCCCACTG-3'. The original binding site of activator protein (AP-1), TGTGAGTCA, was mutated to TCA CTCAGA (Takara Biotechnology Co.). The entire mutated 2,000 bp-length DNA sequence of the miR-146a promoter was produced by Sangon Biotech Co., Ltd.

Western blot analysis. Cells were lysed to extract proteins using radioimmunoprecipitation assay lysis buffer (Beyotime Institute of Biotechnology, Inc.). Protein concentration was determined using Enhanced Bicinchoninic Acid Protein Assay kit (Beyotime Institute of Biotechnology). Extracts containing $50 \mu \mathrm{g}$ total protein were subjected to $10 \%$ sodium dodecyl sulfate-polyacrylamide gel electrophoresis (SDS-PAGE), followed by transfer onto polyvinylidene difluoride membranes (EMD Millipore, Billerica, MA, USA), which were blocked in Tris-buffered saline-0.1\% Tween-20 (TBST) containing $5 \%$ fetal bovine serum (Gibco; Thermo Fisher Scientific, Inc.) for $1 \mathrm{~h}$ at room temperature. The membranes were subsequently probed overnight at $4{ }^{\circ} \mathrm{C}$ with the following primary antibodies: Monoclonal mouse anti-c-jun (1:1,000 dilution; cat no. 2315; Cell Signaling Technology, Inc., Danvers, MA, USA), monoclonal mouse anti-human NF- $\kappa$ B p65 (1:1,000; cat no. 6956; Cell Signaling Technology, Inc.) or monoclonal anti-glyceraldehyde-3-phosphate dehydrogenase (1:1,000 dilution; Cell Signaling Technology, Inc.). Following washing with TBST, membranes were incubated with goat anti-mouse $\operatorname{IgG}$ (1:10,000 dilution; Kangwei Biotech Co.) for $1 \mathrm{~h}$ at room temperature and proteins were visualized by chemiluminescence using an ECL kit (Pierce Biotechnology, Inc., Rockford, IL, USA). Protein expression was quantified using ImageJ (National Institutes of Health, Bethesda, MD, USA).

Nuclear extraction. Nuclear proteins were extracted using a nuclear extraction kit (cat. no. P0028; Beyotime Institute of Biotechnology, Inc.). The cells were washed twice with phosphate-buffered saline (PBS) and lysed in reagent A. 
A

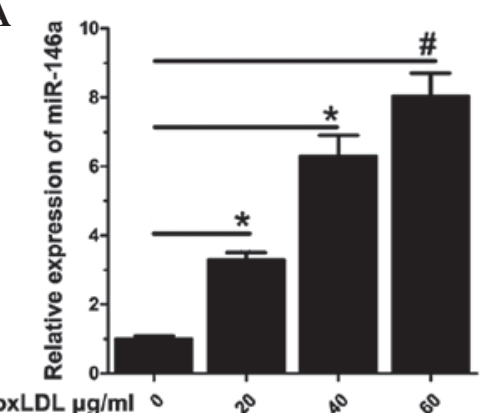

B

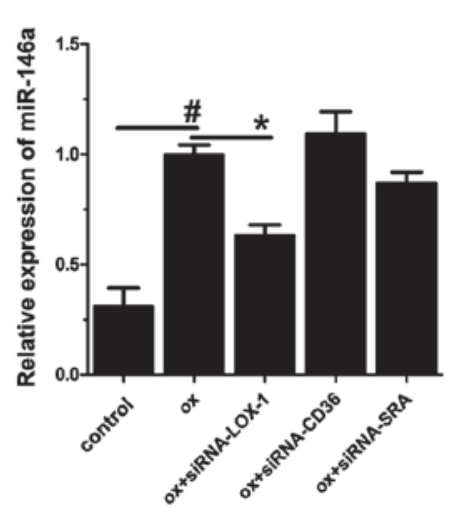

C

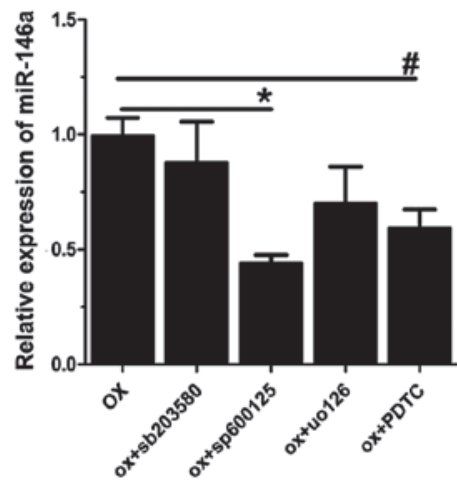

Figure 1. (A) OxLDL dose-dependently enhanced miR-146a expression in THP-1 cells. (B) Inhibition of oxLDL-mediated miR-146a induction by siRNA-mediated knockdown of scavenger receptors LOX-1, SRA and CD36. (C) OxLDL-mediated miR-146a induction was reduced following treatment with c-Jun N-terminal kinase inhibitor SP600125, p38/MAPK inhibitor SB203580, MAPK kinase inhibitor UO126 or nuclear factor $\mathrm{kB}$ inhibitor PDTC. Values are expressed as the mean \pm standard deviation $(\mathrm{n}=5)$. ${ }^{\mathrm{P}}<0.05,{ }^{*} \mathrm{P}<0.01$. oxLDL/ox, oxidized low-density lipoprotein; LOX-1, lectin-like oxLDL receptor-1; SRA, macrophage scavenger receptor class A; PDTC, pyrrolidine dithiocarbamate; siRNA, small interfering RNA; miR, microRNA; MAPK, mitogen-activated protein kinase.
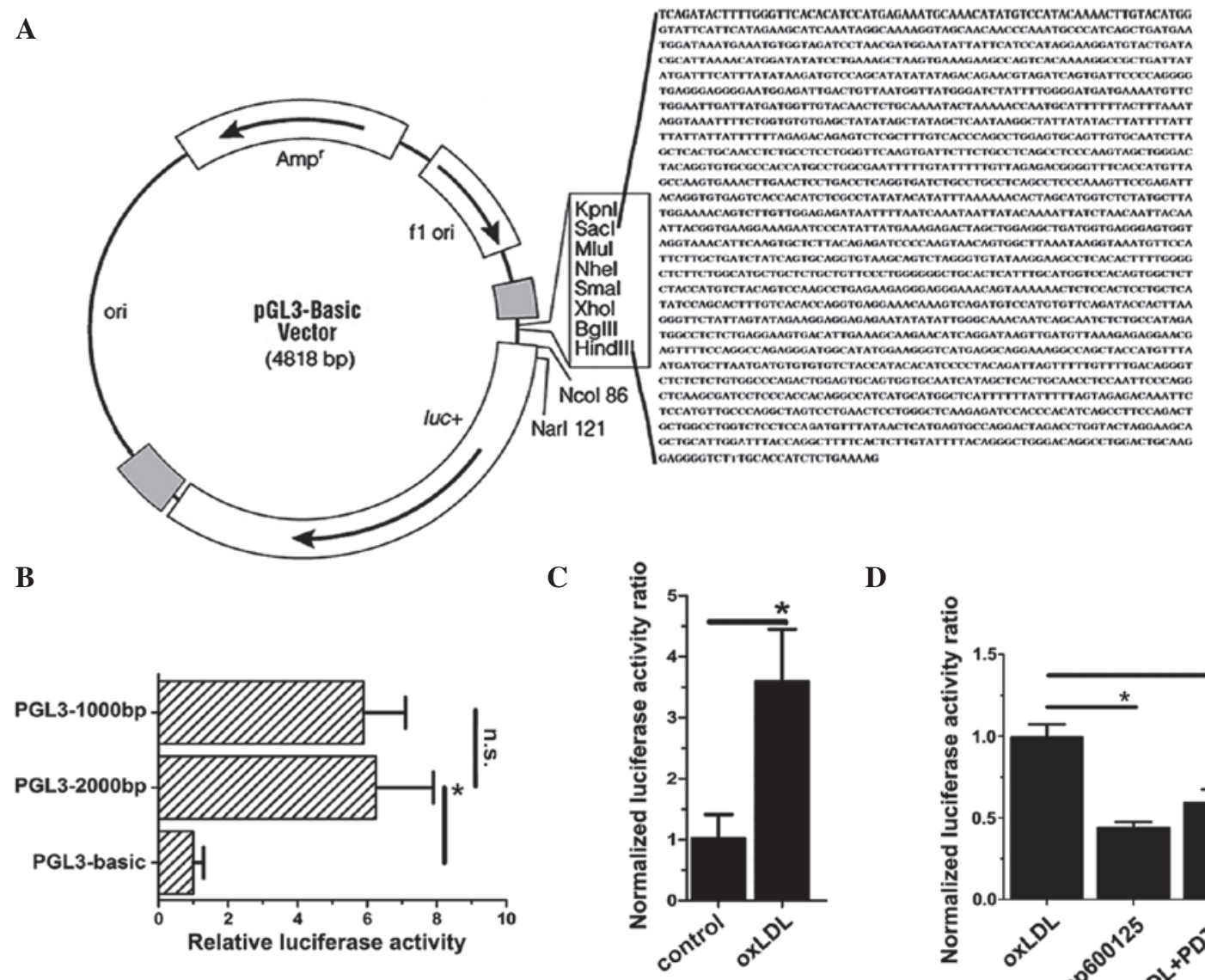

D

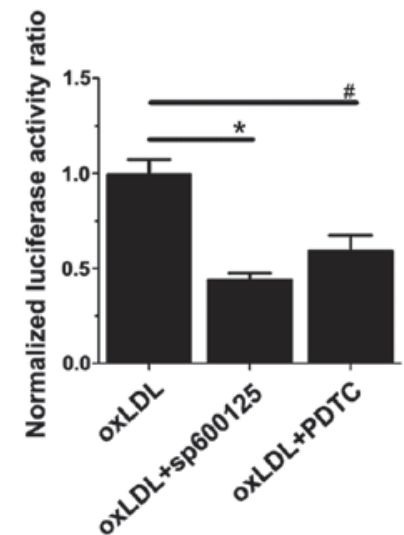

Figure 2. Analysis of miR-146a transcription with luciferase reporter constructs. (A) Fragments of miR-146a were cloned into the PGL3-basic vector. (B) OxLDL promoted transcriptional activation of the reporter driven by a 2,000-bp miR-146a promoter fragment. Truncation of 1,000 bp did not affect promoter activity. (C) oxLDL positively mediated induction of luciferase activity. (D) c-Jun N-terminal kinase inhibitor SP600125 and nuclear factor $\kappa B$ inhibitor PDTC inhibited the oxLDL-mediated induction of luciferase activity. Values are expressed as the mean \pm standard deviation $(\mathrm{n}=5)$. " $\mathrm{P}<0.05,{ }^{\prime \prime} \mathrm{P}<0.01$. PDTC, pyrrolidine dithiocarbamate; miR, microRNA; oxLDL, oxidized low-density lipoprotein; n.s., not significant.

Following vortexing for $5 \mathrm{sec}$, the mixture was incubated on ice for $15 \mathrm{~min}$. Upon addition of reagent B, incubation was continued for $1 \mathrm{~min}$. The lysate was centrifuged at $12,000 \mathrm{xg}$ for $5 \mathrm{~min}$ and the supernatant was discarded. The pellet was lysed by adding the nuclear extraction reagent into the centrifuge tube, vortexing for $15 \mathrm{sec}$ and incubating on ice for $30 \mathrm{sec}$. Following centrifugation for $10 \mathrm{~min}$ at $12,000 \mathrm{x} \mathrm{g}$, the supernatant was collected as the nuclear extract. 


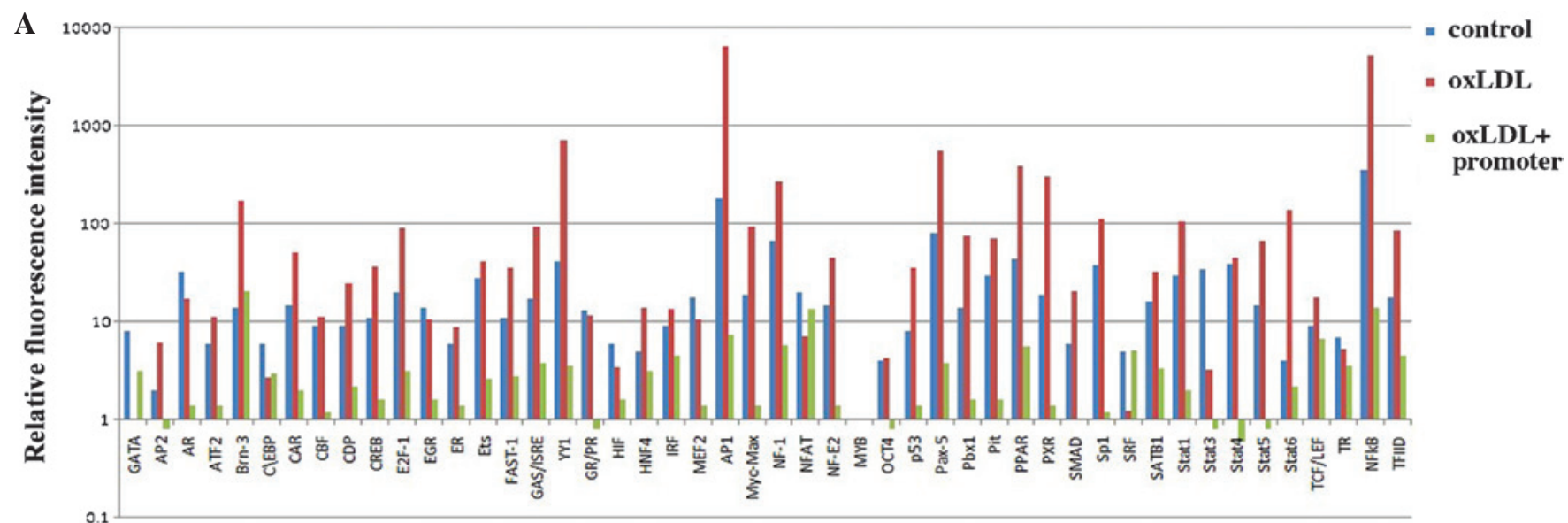

B

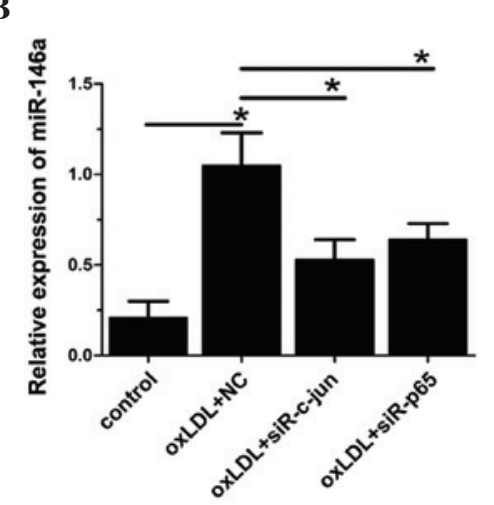

C

...ATATGGAAGGGTCATGAGGCAGGAAAGGCC... ...GAGATTACAGGTGTGAGTCACCACATCTCG... ...GAGACAGAGTCTCGCTTTGTCACCCAGCCT... AP-1 binding sites
D

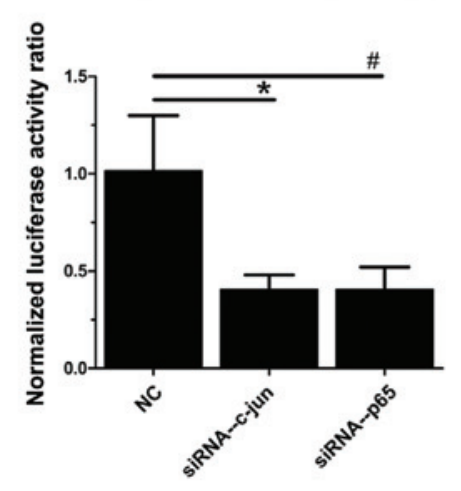

Figure 3. (A) A 'TF promoter binding screening assay' identified AP-1, NF- $\mathrm{B}$ and YY1 as the most potent oxLDL-inducible TFs that bind to the miR-146a promoter. (B) siRNA-mediated knockdown of c-jun or p65 inhibited oxLDL-induced miR-146a expression. (C) Computer-aided prediction of potential AP-1 binding sites within the miR-146a promoter. (D) siRNA-mediated knockdown of c-jun or p65 inhibited oxLDL-induced enhancement of luciferase activity. Values are expressed as the mean \pm standard deviation $(\mathrm{n}=5)$. ${ }^{*} \mathrm{P}<0.05$, ${ }^{\text {}} \mathrm{P}<0.01$. NC, negative control; TF, transcription factor; miR, microRNA; oxLDL, oxidized low-density lipoprotein; siRNA, small interfering RNA; AP-1, activator protein 1.

Transcription factor (TF) filter plate assay. TF detection was performed in nuclear extracts by using the TF filter plate assay kit (cat. no. FA-0010; Signosis, Inc., Santa Clara, CA, USA), according to the manufacturer's instructions. In brief, TF DNA complexes were formed by mixing the TF probe with the nuclear extract. TF/DNA complexes were then separated from free probes by centrifugation at $600 \mathrm{x}$ g. Following hybridization of the TF DNA probe complex with the hybridization plate, the bound probe was detected by chemiluminescence using a luminometer (GloMax ${ }^{\mathrm{TM}} 96$ Microplate Luminometer; Promega Corp.).

Co-immunoprecipitation assay. THP-1 cells were treated with oxLDL $(4 \mu \mathrm{g} / \mathrm{ml})$ for $24 \mathrm{~h}$, harvested and lysed in SDS lysis buffer containing protease inhibitors (Beyotime Institute of Biotechnology). Extracts containing $80 \mu \mathrm{g}$ total protein were incubated with $5 \mu \mathrm{g}$ p 65 antibody (Cell Signaling Technology, Inc.). Following incubation at $4^{\circ} \mathrm{C}$ overnight with agitation, $30 \mu \mathrm{l}$ protein A/G agarose beads (Santa Cruz Biotechnology, Inc., Dallas, TX, USA) were added and samples were incubated with agitation for another $2 \mathrm{~h}$. Following centrifugation for $1 \mathrm{~min}$ at $1,000 \mathrm{x} \mathrm{g}$, agarose beads were washed three times with SDS lysis buffer. Subsequently, $30 \mu 1$ SDS lysis buffer and $15 \mu 12 \mathrm{X}$ SDS-PAGE sample buffer (Beyotime Institute of Biotechnology) were mixed with agarose and the mixture was heated at $100^{\circ} \mathrm{C}$ for $10 \mathrm{~min}$. Following centrifugation at
$1,000 \mathrm{x} g$ for $1 \mathrm{~min}$, supernatants containing the immunoprecipitates were subjected to SDS-PAGE and immunodetection of c-jun and p65 following the previously described western blot protocol.

Chromatin immunoprecipitation assay. Following cross-linking with $1 \%$ formaldehyde in culture medium at room temperature for $10 \mathrm{~min}$, THP- 1 cells $\left(1 \times 10^{7}\right)$ were washed with ice-cold PBS and re-suspended in $1 \mathrm{ml} \mathrm{SDS}$ lysis buffer. DNA fragmentation with an average length of 300-900 nt was achieved by sonication of the lysate, followed by pre-clearing by incubation with $20 \mu \mathrm{l}$ protein A-agarose beads (Beyotime Institute of Biotechnology) for $30 \mathrm{~min}$ at $4^{\circ} \mathrm{C}$. Following removal of the beads by centrifugation (1,000 x g, $1 \mathrm{~min})$, the dissolved chromatin was immunoprecipitated with anti-c-jun antibodies $(2 \mu \mathrm{g})$ overnight at $4^{\circ} \mathrm{C}$ and subsequently incubated with protein A-agarose beads for $1 \mathrm{~h}$. Elution with $150 \mu$ l elution buffer (1\% SDS and $0.1 \mathrm{M} \mathrm{NaHCO}_{3}$ ) provided the immune complex, in which formaldehyde cross-linking was reversed by heating at $65^{\circ} \mathrm{C}$ for $4 \mathrm{~h}$ with $5 \mathrm{M} \mathrm{NaCl}$ and proteinase $\mathrm{K}$ (Beyotime Institute of Biotechnology). Genomic DNA was purified from the immunoprecipitate using a Genomic DNA Isolation kit (Kangwei Biotech Co.) and analyzed by RT-qPCR. The specific primers used were as follows: 1-AP1 forward, 5'-GCGATATCGAGTTATTCCG-3' and reverse, 5'-ACT 
A

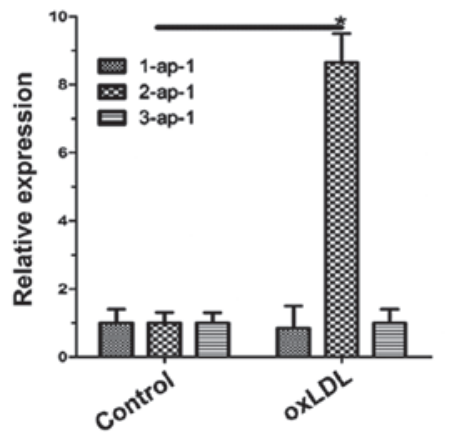

D

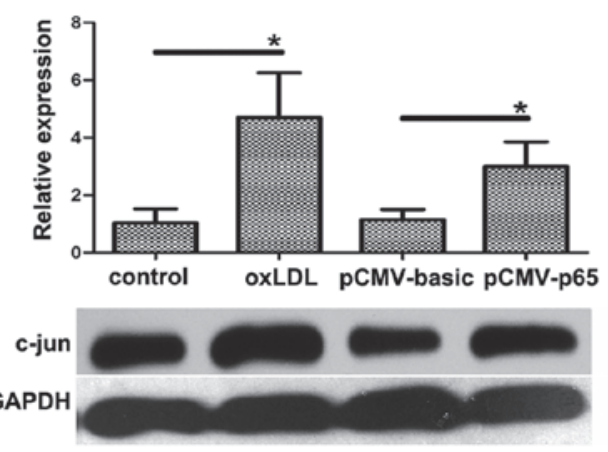

B

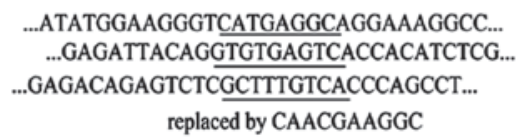
replaced by CAACGAAGGC

$\mathbf{E}$

C

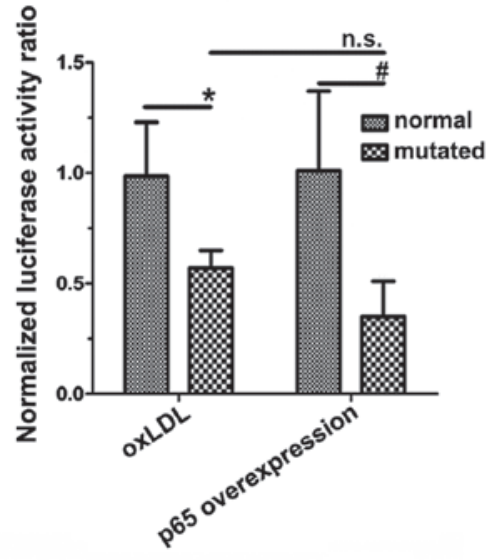

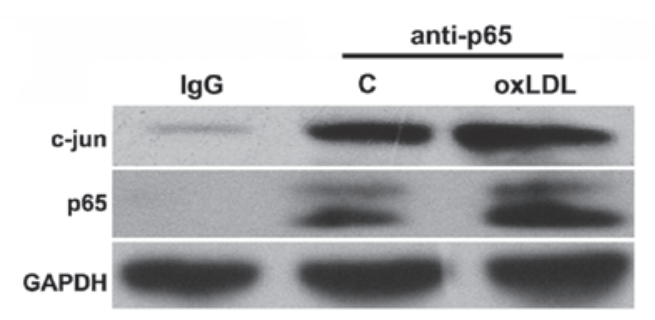

Figure 4. (A) Chromatin immunoprecipitation was performed to precipitate DNA bound with c-jun protein, and primers were designed to amplify the corresponding sequences around the predicted c-jun binding sites on the miR146a promoter. Only the second AP-1 binding site in the miR-146a promoter is functional following oxLDL treatment. (B) The three potential AP-1 binding sites were mutated in the PGL3-mut plasmid (underlined). (C) Luciferase activity of the mutated promoter was reduced and could not be rescued by p65 overexpression. (D) Western blot analysis showed that the protein levels of c-jun in THP-1 cells were increased following oxLDL administration or 065 overexpression. (E) Co-immunoprecipitation assays showed that oxLDL also enhanced the association of $\mathrm{c}$-jun with $\mathrm{p} 65$. Values are expressed as the mean \pm standard deviation $(\mathrm{n}=5)$. ${ }^{*} \mathrm{P}<0.05,{ }^{*} \mathrm{P}<0.01$. pCMV, plasmid cytomegalovirus; oxLDL, oxidized low-density lipoprotein; siRNA, small interfering RNA; AP-1, activator protein 1; IgG, immunoglobulin G; C, control; n.s., not significant.

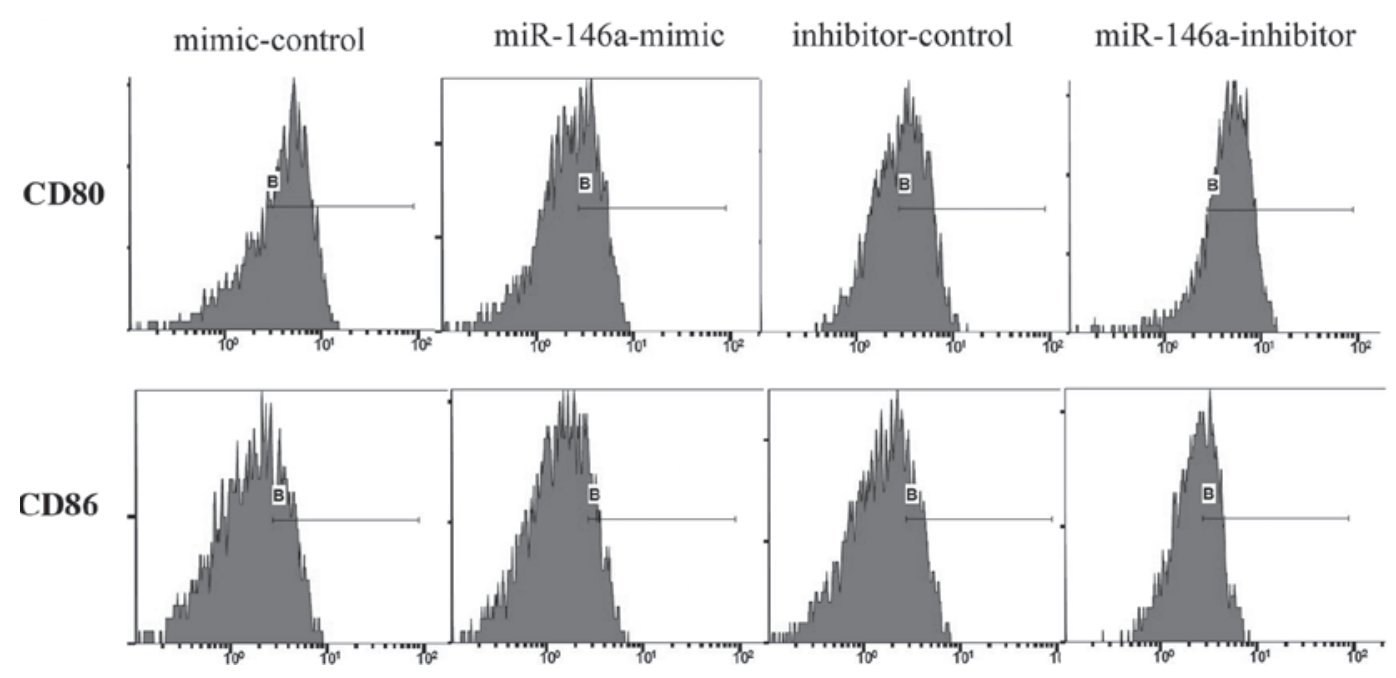

Figure 5. Transfection of a miR-146a-mimic into THP-1 cells decreased the expression of CD80 and CD86, while a miR-146a inhibitor elicited opposite effects. Histograms representative of five experiments are shown. miR, microRNA.

ACAGGTGTGCGCCACCATG-3'; 2-ap1 forward, 5'-TGA TGTCCACACGCGGTGGTAC-3' and reverse; 5'-CACTAG CATGGTCTCTATGC-3'; 3-ap1 forward, 5'-GTCGTTAGA GACGGTATCTACC-3' and reverse, 5'-CACATCCCCTAC AGATTAG-3'.

Flow cytometry. THP-1 cells were transfected with miR-146a mimic/mimic-control or miR-146a inhibitors/inhibitor-control for $24 \mathrm{~h}$. Following two washes with PBS, cells were incubated with monoclonal mouse anti-human CD86 (1:1,000; cat no. 563411; BD Biosciences, Franklin Lakes, CA, USA) or monoclonal mouse anti-human CD80 antibodies (1:1,000; cat no. 560296; BD Biosciences). Finally, analysis was performed using an FC500 flow cytometer (Beckman Coulter, Inc., Brea, CA, USA) with CellQuest Pro software (BD Biosciences, Inc.). 
Bioinformatics analysis. Computer-aided prediction of potential AP-1 binding sites within the miR-146a promoter was conducted using the online transcription factor search software TFSearch (www.cbrc.jp; Computational Biology Research Consortium, Tokyo, Japan). The 2,000 bp promotor segment of miR-146a was used to search for the potential AP-1 binding sites.

Statistical analysis. Values are expressed as the mean \pm standard deviation. Differences were compared using the t-test and analysis of variance using SPSS software, version 19.0 (IBM SPSS, Armonk, NY, USA). P<0.05 was considered to indicate a statistically significant difference. All experiments were performed at least three times.

\section{Results}

oxLDL enhances miR-146a expression via LOX-1 and the JNK pathway. In order to explore the mechanism of oxLDL-mediated regulation of miR-146a, THP-1 cells were treated with various doses of oxLDL. As shown in Fig. 1A, oxLDL increased the levels of miR-146a in a concentration-dependent manner. Since oxLDL is mainly ingested by macrophages through scavenger receptors (14), THP-1 cells were then treated with siRNAs with specificity for SRA, CD36 or LOX-1 prior to incubation with oxLDL. Of note, LOX-1 knockdown significantly reduced the induction of miR-146a expression by oxLDL $(\mathrm{P}<0.05)$ (Fig. 1B). Since oxLDL is known to activate the MAPK pathway, its possible involvement was then assessed by pre-incubation with chemical inhibitors of p38, JNK and ERK1/2 for 30 min prior to administration of oxLDL. miR-146a expression was significantly deceased $(\mathrm{P}<0.05)$ when JNK was inhibited by SP600125 (Fig. 1C). In addition, PDTC, a known inhibitor of NF- $\kappa \mathrm{B}$, also decreased expression of miR-146a $(\mathrm{P}<0.05)$ (Fig. 1C). These results suggested important functions of JNK and NF- $\mathrm{B}$ in oxLDL-mediated activation of miR-146a.

oxLDL stimulates miRNA-146a promoter activity. To address whether oxLDL activates miR-146a via a transcriptional mechanism, a dual luciferase reporter assay was performed. Reporter plasmid constructs driven by fragments of the miR-146a promoter were generated (Fig. 2A) and their activity was analyzed. As shown in Fig. 2B, oxLDL potently induced luciferase activity of a plasmid driven by the 2,000-bp miR-146a promoter fragment. Truncation of the promoter by $1,000 \mathrm{bp}$ did not impair this activity, suggesting that the first $1,000 \mathrm{bp}$ upstream of the transcription start site are crucial for the regulation of miR-146a transcription by oxLDL. Of note, pre-treatment of THP-1 cells with either SP600125 or PDTC abrogated the induction of luciferase activity by oxLDL (Fig. 2C). These findings demonstrated that oxLDL promotes the transcriptional activation of miR-146a indirectly.

Transcriptional activation of $m i R-146 a$ by oxLDL requires $A P-1$ and $N F-\kappa B$. To further explore the mechanism of the regulation of miR-146a transcription, the 'TF promoter binding screening assay' was utilized. As shown in Fig. 3A, AP-1, NF- $\kappa$ B and YY1 were identified as the most prominent oxLDL-inducible transcription factors that bind to the miR-146a promoter. To validate this finding, THP-1 cells were transfected with siRNA against c-jun, a component of the AP-1 transcription factor complex, or against the $\mathrm{p} 65$ sub-unit of $\mathrm{NF}-\kappa \mathrm{B}$ prior to oxLDL treatment. In addition, RT-qPCR analysis showed that knockdown of either c-jun or p65 decreased miR-146a expression (Fig. 3B). Bioinformatics analysis identified three potential AP-1 binding sites within the miR-146a promoter (Fig. 3C). Furthermore, siRNA-mediated knockdown of c-jun was shown to decrease the luciferase activity of a miR-146a promoter-driven reporter plasmid (Fig. 3D). Similar results were obtained by siRNA-mediated p65 knockdown (Fig. 3B and D). These results indicated a participation of c-jun and p65 in oxLDL-induced miR-146a transcription.

Mutation of the AP-1 binding site reduces the activation of miRNA-146a transcription by oxLDL and $p 65$. Chromatin immunoprecipitation was performed to validate the bioinformatics prediction of the presence of AP-1 binding sites within the miRNA-146a promoter. As shown in Fig. 4A, only the second of the three potential AP-1 binding sites was occupied by c-jun. Next, a luciferase reporter construct driven by a miRNA-146a promoter bearing a mutation in the functional AP-1 binding site was generated (Fig. 4B). After oxLDL treatment, the activity of plasmids driven by the mutated promoter sequence was significantly reduced compared to that of the plasmid carrying the wild-type sequence $(\mathrm{P}<0.05)$ (Fig. 4C). Plasmid-mediated overexpression of p65 did not reverse this effect (Fig. 4C). Furthermore, western blot analysis showed that oxLDL treatment as well as forced expression of p65 led to increases in c-jun levels (Fig. 4D). Thus, p65 appears to induce the miR-146a promoter via AP-1. In addition, a co-immunoprecipitation assay showed increased binding of c-jun to p65 following treatment of THP-1 cells with oxLDL (Fig. 4E). These results showed that oxLDL stimulation promotes the binding of p65 to c-jun.

miR-146a suppresses macrophage maturation. To explore the function of miR-146a in macrophages, the present study assessed the expression of the maturation markers CD80 and CD86 in THP-1 cells. Transfection with a miR-146a-mimic decreased the expression of CD80 and CD86 expression. Conversely, transfection with a miR-146a inhibitor reversed this effect (Fig. 5). These results suggested that miR-146a suppresses macrophage-associated antigen presentation by targeting CD80 and CD86, in consistency with the findings of a previous study by our group (12).

\section{Discussion}

The present study explored the regulatory mechanisms of miR-146a expression by oxLDL in macrophages. The scavenger receptor-LOX-1, the JNK pathway and transcription factors, including c-jun and p65, were demonstrated to be involved in miR-146a transcription. Previous studies have identified miRNAs146a as a marker for the prediction of clinical responses and as a potential therapeutic target in hepatocellular carcinoma, interferon (IFN)-based therapy (15) and castration-resistant prostate cancer (16). Loss of endogenous miR-146a is critical for overexpression of epidermal growth factor receptor and administration of synthetic miR-146a may be of therapeutic 
value in suppressing breast-cancer metastasis $(17,18)$. In addition, miR-146a is a negative regulator of the IFN pathway, thereby representing a potential therapeutic for the interventions of lupus (19). miR-146a stimulates innate immunity to evade re-current bacterial infection (4) and has been shown to have a crucial role in monocytic cell-based endotoxin-induced cross-tolerance in vitro (20). Hepatitis C virus infection may repress the induction of miR-146a by interfering with TLR-7 signaling (21). miR-146a may also function as a negative feedback regulator of lipopolysaccharide-induced production of inflammatory cytokines (22). Overexpression of miR-146a represents a potential strategy for exogenous modulation of immune tolerance during microbial infection and sepsis (23). Microenvironmental factors are important for JNK and NF- $\mathrm{BB}$ activation in inflammation-driven diseases (24) and miR-146a has been shown to act as a tumor-suppressor by inhibiting the expression of tumor-promoting cytokines and growth factors via the $\mathrm{NF}-\kappa \mathrm{B}$ pathway . miR-146a negatively regulates the release of interleukin-8 and RANTES in human alveolar epithelial cells (25). Despite the well-documented involvement of miR-146a in multiple immune-inflammatory diseases, the mechanism by which miR-146a regulates atherosclerosis has largely remained elusive.

A previous study by our group showed that miR-146a regulates the maturation process of macrophages and their pro-inflammatory cytokine secretion by targeting CD40 L in oxLDL-stimulated dendritic cells (12). However, the mechanism of the stimulation of miR-146a expression by oxLDL has remained elusive. The present study revealed that oxLDL promotes miR-146a induction via the scavenger receptor-LOX-1 as well as the JNK pathway. Furthermore, NF- $\kappa \mathrm{B}$ also regulated miR-146a expression induced by oxLDL. The transcription factors c-jun and p65 were demonstrated to participate in miR-146a transcription. However, chromatin immunoprecipitation experiments showed that p65 enhanced miR-146a transcription through increasing c-jun The formation of a complex between p65 and c-jun promoted miR-146a trancription. By providing a mechanism of the stimulation of miR-146a expression in response to oxLDL treatment, the present study enhanced the current understanding of the complex physiology of atherosclerosis. However, as indicated by the TF promoter binding screening assay, further transcription factors are involved in miR-146a transcription. Their exact roles as well as any possible association with c-jun remain to be elucidated.

In addition, the present study addressed the role of miR-146a in macrophage maturation. Flow cytometry results showed that miR-146a suppressed the expression of CD86 and CD80 in THP-1 cells, which indicated an important immunological function of miR-146a. Previous studies showed that miR-146a inhibits the secretion of inflammatory factors through the JNK and $\mathrm{NF}-\kappa \mathrm{B}$ pathways. In the present study, JNK and $\mathrm{NF}-\kappa \mathrm{B}$ were shown to mediate miR-146a transcription induced by oxLDL. The crosstalk between miR-146a and the JNK and $\mathrm{NF}-\kappa \mathrm{B}$ pathways requires further elucidation. The exploration of additional roles of miR-146a in macrophages as well as the mechanism by which miR-146a modulates the development of atherosclerosis are currently underway in our group.

In conclusion, the present study demonstrated that oxLDL promotes the formation of a complex between p65 and c-jun in macrophages, which in turn binds to the miRNA-146a promoter and activates its transcription. Furthermore, miR-146a was shown to suppress the expression of macrophage maturation markers. The present study may represent a basis for the development of novel therapeutic targets for the treatment of atherosclerosis.

\section{Acknowledgement}

The present study was funded by the Natural Science Foundation of China (grant nos. 81200214/H0215 and 81400205).

\section{References}

1. Libby P, Ridker PM and Hansson GK: Progress and challenges in translating the biology of atherosclerosis. Nature 473: 317-325, 2011.

2. Han J, Lee Y, Yeom KH, Nam JW, Heo I, Rhee JK, Sohn SY, Cho Y, Zhang BT and Kim VN: Molecular basis for the recognition of primary microRNAs by the Drosha-DGCR8 complex. Cell 125: 887-901, 2006.

3. Bartel DP: MicroRNAs: Genomics, biogenesis, mechanism, and function. Cell 116, 281-297, 2004.

4. Nahid MA, Pauley KM, Satoh M and Chan EK: MiR-146a is critical for endotoxin-induced tolerance: Implication in innate immunity. J Biol Chem 284: 34590-34599, 2009.

5. Yang K, He YS, Wang XQ, Lu L, Chen QJ, Liu J, Sun Z and Shen WF: MiR-146a inhibits oxidized low-density lipoprotein-induced lipid accumulation and inflammatory response via targeting toll-like receptor 4. FEBS Lett 585: 854-860, 2011.

6. Hou J, Wang P, Lin L, Liu X, Ma F, An H, Wang Z and Cao X: MicroRNA-146a feedback inhibits RIG-I-dependent Type I IFN production in macrophages by targeting TRAF6, IRAK1 and IRAK2. J Immunol 183: 2150-2158, 2009.

7. Tang Y, Luo X, Cui H, Ni X, Yuan M, Guo Y, Huang X, Zhou H, de Vries N, Tak PP, et al: MicroRNA-146A contributes to abnormal activation of the type I interferon pathway in human lupus by targeting the key signaling proteins. Arthritis Rheum 60: 1065-1075, 2009.

8. Pauley KM, Satoh M, Chan AL, Bubb MR, Reeves WH and Chan EK: Upregulated miR-146a expression in peripheral blood mononuclear cells from rheumatoid arthritis patients. Arthritis Res Ther 10: R101, 2008.

9. Nakasa T, Miyaki S, Okubo A, Hashimoto M, Nishida K, Ochi M and Asahara H: Expression of microRNA-146 in rheumatoid arthritis synovial tissue. Arthritis Rheum 58: 1284-1292, 2008.

10. Stanczyk J, Pedrioli DM, Brentano F, Sanchez-Pernaute O, Kolling C, Gay RE, Detmar M, Gay S and Kyburz D: Altered expression of microRNA in synovial fibroblasts and synovial tissue in rheumatoid arthritis. Arthritis Rheum 58: 1001-1009, 2008.

11. Sonkoly E, Stahle M and Pivarcsi A: MicroRNAs: Novel regulators in skin inflammation. Clin. Exp Dermatol 33: 312-315, 2008.

12. Chen T, Li Z, Jing T, Zhu W, Ge J, Zheng X, Pan X, Yan H and Zhu J: MicroRNA-146a regulates the maturation process and pro-inflammatory cytokine secretion by targeting CD40L in oxLDL-stimulated dendritic cells. FEBS Lett 585: 567-573, 2011.

13. Subramanian M and Tabas I: Dendritic cells in atherosclerosis. Semin Immunopathol 36: 93-102, 2014.

14. Murphy JE, Tedbury PR, Homer-Vanniasinkam S, Walker JH and Ponnambalam S: Biochemistry and cell biology of mammalian scavenger receptors. Atherosclerosis 182: 1-15, 2005.

15. Tomokuni A, Eguchi H, Tomimaru Y, Wada H, Kawamoto K, Kobayashi S, Marubashi S, Tanemura M, Nagano H, Mori M and Doki Y: MiR-146a suppresses the sensitivity to interferon- $\alpha$ in hepatocellular carcinoma cells. Biochem Biophys Res Commun 414: 675-680, 2011.

16. Xu B, Wang N, Wang X, Tong N, Shao N, Tao J, Li P, Niu X, Feng N, Zhang L, et al: MiR-146a suppresses tumor growth and progression by targeting EGFR pathway and in a p-ERK-dependent manner in castration-resistant prostate cancer. Prostate 72: 1171-1178, 2012.

17. Bhaumik D, Scott GK, Schokrpur S, Patil CK, Campisi J and Benz CC: Expression of microRNA-146 suppresses NF-kappaB activity with reduction of metastatic potential in breast cancer cells. Oncogene 27: 5643-5647, 2008. 
18. Hurst DR, Edmonds MD, Scott GK, Benz CC, Vaidya KS and Welch DR: Breast cancer metastasis suppressor 1 up-regulates miR-146, which suppresses breast cancer metastasis. Cancer Res 69: 1279-1283, 2009.

19. Tang Y, Luo X, Cui H, Ni X, Yuan M, Guo Y, Huang X, Zhou H, de Vries N, Tak PP, et al: MicroRNA-146A contributes to abnormal activation of the type I interferon pathway in human lupus by targeting the key signaling proteins. Arthritis Rheum 60: 1065-1075, 2009.

20. Nahid MA, Satoh M and Chan EK: Mechanistic role of microRNA-146a in endotoxin-induced differential cross-regulation of TLR signaling. J Immunol 186: 1723-1734, 2011.

21. El-Ekiaby N, Hamdi N, Negm M, Ahmed R, Zekri AR, Esmat G and Abdelaziz AI: Repressed induction of interferon-related microRNAs miR-146a and miR-155 in peripheral blood mononuclear cells infected with HCV genotype 4. FEBS Open Bio 2: 179-186, 2012.
22. He Y, Sun X, Huang C, Long XR, Lin X, Zhang L, Lv XW and $\mathrm{Li} \mathrm{J}$ : MiR-146a regulates IL-6 production in lipopolysaccharide-induced RAW264.7 macrophage cells by inhibiting Notch1. Inflammation 37: 71-82, 2014.

23. Quinn EM, Wang JH, O'Callaghan G and Redmond HP: MicroRNA-146a is upregulated by and negatively regulates TLR2 signaling. PLoS One 8: e62232, 2013.

24. Crone SG, Jacobsen A, Federspiel B, Bardram L, Krogh A, Lund AH and Friis-Hansen L: microRNA-146a inhibits $\mathrm{G}$ protein-coupled receptor-mediated activation of NF- $x \mathrm{~B}$ by targeting CARD10 and COPS8 in gastric cancer. Mol Cancer 11: 71, 2012.

25. Perry MM, Williams AE, Tsitsiou E, Larner-Svensson HM and Lindsay MA: Divergent intracellular pathways regulate interleukin-1beta-induced miR-146a and miR-146b expression and chemokine release in human alveolar epithelial cells. FEBS Lett 583: 3349-3355, 2009. 